\title{
Source Identification of Videos Transmitted in Lossy Wireless Networks
}

\author{
Jagbeer Kaur ${ }^{1}$, Deep Kamal Kaur Randhawa ${ }^{1}$ \\ ECE Department, GNDU RC Jalandhar, India ${ }^{1}$
}

\begin{abstract}
The sensor pattern noise based method exploits the inherent fingerprint of the camera sensor and is universally applicable. This method has the best performance reported so far among the existing methods and only method effective in lossy wireless network incorporating wireless channel characteristics and protection from security threats like blurring, blocking and spoofing attack. Experiment test was conducted with 20 test each on 8 videos of eight wireless cameras taking randomly 5 frames of the video to be tested using Sensor Pattern Noise based on correlation based coeffient matching and our proposed work based on Gaussian Mixture Model matching. It leads us to conclusion that it is more reliable, accurate and less Complex. It is applicable on different size video, requirement of random frames is very less as compared to existed work and help to create trained data set only once.
\end{abstract}

Keyword: correlation coefficient, Gaussian mixture models, sensor pattern noise.

\section{INTRODUCTION}

There are two categories of video source identification. The first category is device class identification which is used to tell the manufacturer or model of the device. The Second Category is specific device identification which deals to identify the individual device that has produced the data. The image source identification methods can be easily adopted directly for video source identification. It is bit easier to distinguish different models but difficult when identifying specific device. Some methods like watermarking technique helps to find specific device but have special limits on videos.

Now, with advancement of technology, the world enters in wireless communication. The use of wireless camera has increased. The wireless video cameras are generally used in a special security region. These cameras have no local storage. The video is captured and wirelessly streamed to a sink. Due to transmission delay in wireless streaming, some packets are lost causing blocking and blurring appear in the received frames. Thus, when working on wireless networks the concern is to authenticate source of video. There is need to exploits the inherent fingerprint of camera sensor which must be universally applicable.

Video Source identification is widely used for validating video evidence in court as it necessary to prove that the video was truly recorded by the suspected camera [1]. It also, helps in tracking down video piracy crimes [2] [3] and help to regulate individual video sources on internet where videos are shared at large scale [4].

Modern digital cameras use tag such as date and time, camera settings, or the serial number of the camera when producing the image. Currently, there is no standard metadata pattern for video files. Also, when this metadata is present, it can be easily removed or manipulated. This can lead us to doubt about the source of image. Another method is Defective Pixels identification in which the defective pixels can act as a digital finger print which are present in sensor [5]. Defective pixels can be corrected by modern camera with additional features. This will give us false result and our motive to authenticate the suspect camera will be not accomplished.

Researcher moves to new techniques which focus on individual pixels that may report slightly lower or higher values than their neighbors. The Method based on PRNU (Photo Response Non-Uniformity) which is the output signals from pixels produced when the illumination incident on a number of pixels is exactly same for all pixels.But PRNU method suffers from performance degradation when the video is captured by wireless camera and contain blocking and blurring during transmission. Besides, most of these sensor methods are computationally expensive and are not suitable for fast identification. There will be need of making large data base. Recent work focus on fixed pattern extraction. Sensor pattern noise has been reported with best performance so far. It is based on idea that correlated signal is compressible and predictable but uncorrelated noise is not. This means each sensor has its own unique noise pattern. Sensor pattern noise which is extracted from a frame of video will be tested and compared with the suspected patterns from the trained database of cameras. When the two patterns are highly similar, almost having negligible difference than this indicates source might be same. Thus, it helps in source identification. 


\section{International Journal of Innovative Research in Electrical, Electronics, Instrumentation and Control Engineering}

\section{ISO 3297:2007 Certified}

Vol. 5, Issue 5, May 2017

Wireless cameras have no storage and mostly used in surveillance system. They capture a video and wirelessly streamed to the sink. Consider sensor resolution as video resolution because generally during on boarding process more sophisticated cameras improve the video quality. This means video distortion will be caused during wireless transmission. Thus, Packet loss during transmission cause blocking and blurring. Video blocking occurs when data of some blocks are missing. Video blurring is result of high compression ratio. It is due to losing high frequency component. During transmission delay this high frequency component is lost due to packet loss which leads to missing of blocks. As discussed in literature survey methods are for post-mortem forensics analysis and high computational cost is needed. Sensor Pattern Noise method is the most effective method so far. The technique takes approx. 10 seconds for a video frame of $640 \times 480$ on simple Intel Core 2 Duo CPU. It needs approximately 200 frames to make reliable decision. It is too long time to detect spoofing attack and it is a security threat which needs concern. Spoofing attack means that an attacker had send another video to the sink using the victim's identity. Sensor Pattern Noise also deals with this issue easily. Source Identification when working in lossy wireless network is active topic of research and need to explored more to make it much faster with less complexity instead of the post-mortem solutions provided by the existing method. Below is literature review regarding the research work.

\section{LITERATURE REVIEW}

The research on video source identification is quite similar to image source identification, and often similar techniques are used for both identifications. A brief survey has been paragraphed below after reading the main outcomes of various journals on this topic. Kharrazi et al. [7] had proposed a simple method to identify the camera model which was used to obtain an image by distinguishing between images captured by a two using color image features like average pixel values and RGB correlation factor with SVM to identify device which have captured the image. Result shows acceptable accuracy even when the image is re-compressed. Similarly, Celiktutan et al. [8] focused on problem to identify a source cell-phone in blind manner by differentiating the color characteristic which gives footprints in form of correlation factor across the adjacent pixel of the images. For this purpose, they explored various classifier which deals with similar image quality measures and high order wavelet statistics. They had used SVM and KNN classifiers to detect the cell-phone. Choi et al. [9] further included lens radial distortions as part of the features extraction. By using lenses with spherical surface having inherent radial distortion gives unique fingerprints of the images and also help in reducing manufacturing cost. Popescu et. al. [10] deals to authenticate an image by finding digital tampering in absence of digital signature or water marking. They have developed a detection tool for forensics using the Expectation/Maximization algorithm to identify the camera patterns, based on which different image sources are classified. A large database of 200 gray scale images was built and pattern was searched using Fourier transform from the original unadulterated images. The periodic patterns obtained during re-sampling using Fourier domain can help in telling the model or the manufacturer of the device, instead of identifying the individual camera that produced the image for forensic applications. Geradts et al. [5] proposed to utilize sensor dead pixels or hot pixels in identification of specific image source. They examined and conclude that noise levels should be used for investigation because the camera of suspect can help to give a fixed pattern noise even if we have nothing in it. Lukas et al. [6] also proposed to examine a suspected camera using sensor pattern noise. The first need is to determine reference pattern noise. This will give a unique fingerprint of each camera for identification. They have considered the reference pattern noise as a spread spectrum watermark established using a correlation detector. They concluded that sensor pattern noise estimation using wavelet decomposition can distinguish between two cameras of same brand also. Li et. al. [11] proposed source identification using sensor pattern noise method. It leads us to digital wavelet transform domain to enhance even the weak component of the pattern noise.

Kurosawa et al. [12] propose that source identification can be achieved by measuring the dark current noise of the sensor which is used to give the device fingerprint. It is effective for dark current noise extracted from 100 dark frames. Chen et al. [2] inherited the idea proposed in [13] and applied it to videos. They also investigate the problem of determining whether two video clips came from the same camcorder and the problem of whether two differently transcoded versions of one movie came from the same camcorder. The source identification technique is based on estimating the Maximum Likelihood factor and deals with blocking and blurring using PRNU normalized crosscorrelation. With decrease in video quality, there was need to increase video length that means will always require large data set and accuracy decreases with video quality. This is a disadvantage of using PRNU pattern noise.

Houten et al. [14] and Hyun et al. [15] extended Chen's method to implement on low resolution videos. It is based on enhanced PRNU pattern noise method. All these solutions also suffer from performance degradation when the video is captured by wireless cameras because will contain blocking and blurring. These methods are not only computationally expensive but not also suitable for fast identification. In present time, use of wireless cameras has increased. They are widely used for security purpose. The security issues like spoofing attack are stemming in the wireless communication. 


\section{ISO 3297:2007 Certified}

Vol. 5, Issue 5, May 2017

This means need faster and most accurate method to be explored. Mehrish A et. al. [16] has proposed that Non -linear transformation affects in calculation of PRNU noise. Thus, Raw values plays an important role. They can be used in Maximum Likelihood Estimation. The results were effective for small dimension patches. The raw data is not available readily, so they have estimated it using poisson process. The PRNU is estimated using Maximum Like li-hood Estimation Technique. Lin X et. al. [17] has proposed the PRNU noise component as the main component of sensor pattern noise. It can be enhanced using Filtering of Distortion. The paper put forward to work on dimensionality reduction method as the proposed method process is much more complicated.

Lawgaly A et. al. [18] has concentrate on different color channels for PRNU estimation. They have used Locally Adaptive DCT filtering and Weighted Averaging value of three color channels. The weighted average value of the three channel helps to eliminate the residual noise for best match. It is complex technique which requires high time value, not suitable in wireless network for fast detection. Also, require large dataset to train and test a query video. Duan Y. et. al. [19] has proposed identification of CT scanner. The first aim was to identify original sensor pattern noise. The second aim was to find a system to reconstruct of 3D image from sensor pattern noise of test image to find the parameters which vary. The parameter was correlation values. The work concludes to focus on different image denoising filter. Fogie et. al. [20] and J. Bellardo [21] has discussed several possible cases of wireless cameras attack. They have also discussed the substitution and interception attack. However, they did not provide any solution.

Pande A. et. al. [22] described the hardware architecture for video Authentication using sensor pattern noise and with fellow member S. Chen has proposed an efficient method for real-time applications. S. Chen et. al. [1] proposed sensor pattern noise method is efficient method. It works effectively for identifying wirelessly transmitted videos which have blocking and blurring due to packet loss during wireless transmissions. In addition, they proposed to incorporate selective frame processing and wireless channel signatures in source identification, which makes source identification faster. Extensive real-world experiments were conducted to validate the method. The results show that the accuracy of source identification based on the proposed method is far better than the existing methods in the presence of video blocking and blurring. The method identifies the video source in a real-time environment to detect spoofing attack. The paper has conclusion that number of existing studies are orthogonal to source identification. So, there is need to explore and incorporate it with the method to make it more accurate and less complex.

\section{VIDEO SOURCE IDENTIFICATION USING SENSOR PATTERN NOISE}

Digital recording device such as digital camera adopts various type of digital image sensors. The digital sensor can be made up of a charge-coupled device (CCD) or complementary metal-oxide-semiconductor (CMOS) or a junction fieldeffect transistor (JFET). The digital image sensor has large number of photon detectors. These photon detectors work on the photoelectric effect. The Photon detectors convert photoelectrons into electrical signals. The strength of the electrical signal depends on the photon detectors sensitivity towards light. The light sensitivity of the photon detectors varies slightly. This is due to the imperfections created during the manufacturing process of the silicon which is used to form the photon detectors. The different uncorrelated multiplicative pattern noise is generated by the difference in sensitivity of light by each pixel. Consequently, every digital sensor cast a unique sensor pattern noise (SPN) onto images (frames) it takes. The SPN act as a sensor fingerprint that identifies a source digital imaging device. Number of methods has been proposed in literature in which correlation of pattern noise of frames was used for identification of source cameras. The SPN quality is first estimated which will be used for reference. It can be obtained by capturing uniform images such as blue sky images. The SPN of the test image will be estimated. Correlation value will be calculated between the reference SPN and test SPN. If the calculated correlation value is higher than a specified threshold. The Test image is surely captured from the digital camera of reference SPN. The drawback of this method is that It is little bit complex as a large number of frames are required and their pattern noises need to be correlated for better accuracy rates. In this work, GMM (Gaussian Mixture Model) based classifier has been proposed which found to be more effective than correlation based method. The proposed framework consists of frame extraction phase, feature extraction phase, feature training phase and finally the classification phase in which test frames are classified according to their source cameras identified by classifier.

A. System module for feature extraction phase

In this module sensor pattern noise has been evaluated for randomly selected camera frames as shown in figure 3.1 .

B. System module for classification or identification phase

In this module, identification of source has been carried out using Gaussian mixture models. A brief of the steps involved are described as shown in figure 3.2. 


\section{International Journal of Innovative Research in}

Electrical, Electronics, Instrumentation and Control Engineering

\section{ISO 3297:2007 Certified}

Vol. 5, Issue 5, May 2017

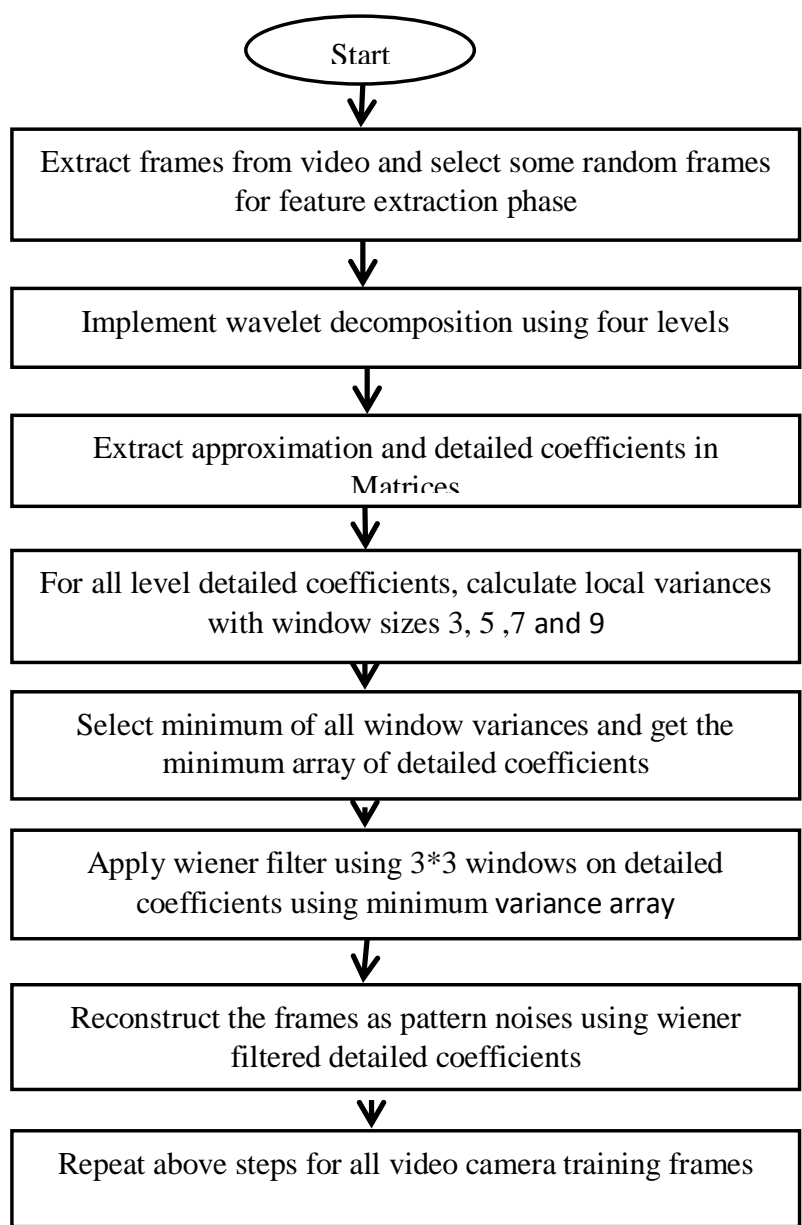

Figure 3.1 Flowchart for pattern noise generation

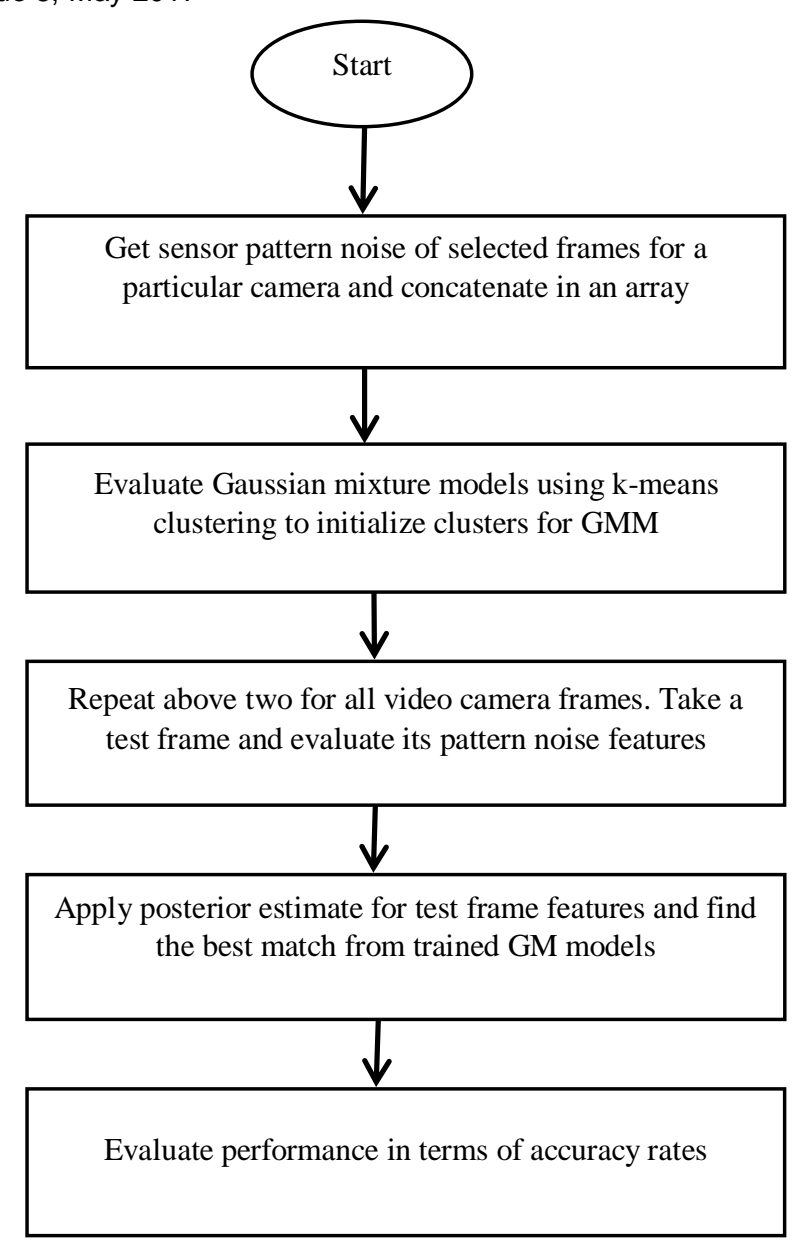

Figure 3.2 Steps in detail for proposed algorithm

\section{Video Data}

Video data has been collected for eight digital cameras used in wireless transmission. Table below shows the video cameras chosen in this work.

Table 3.1 A list of video cameras selected for testing

\begin{tabular}{|l|}
\hline AXIS M1011 \\
\hline D-Link DCS-942L \\
\hline Foscam FI8910W \\
\hline Smiledrive Panoramic 360 \\
\hline TP-Link NC220 \\
\hline TRENDnet TV-IP672W \\
\hline WVC80N Camera test \\
\hline ZVision AHD 1.3 MP \\
\hline
\end{tabular}

2. Frame making and gray-scale conversion

In this step, frames have been extracted from the video using VideoReader Mat lab command. VideoReader (filename) creates object $\mathrm{v}$ to read video data from the file named filename. To decrease complexity, only gray-scale image has been used for sensor pattern noise generation. Now there is only one channel for further processing. For this rgb2gray Mat lab function has been used.

3. Discrete wavelet implementation for sensor pattern noise estimation

The essential methodology [23] to wavelet based image re-construction has three main steps. From these first and third steps has been chosen as it is but second step has been modified using the local variance estimation and wiener filtering. The steps involved in this are described as below 


\section{International Journal of Innovative Research in} Electrical, Electronics, Instrumentation and Control Engineering

\section{ISO 3297:2007 Certified}

Vol. 5, Issue 5, May 2017

a) Compute the two-dimensional wavelet transform of the frame using debauchee's wavelet.

b) For every level, do steps c) and d). For one fixed level, we denote the horizontal, vertical, and diagonal subbands as $\mathrm{h}(\mathrm{i}, \mathrm{j}), \mathrm{v}(\mathrm{i}, \mathrm{j})$ and $\mathrm{d}(\mathrm{i}, \mathrm{j})$ where $(\mathrm{i}, \mathrm{j})$ runs through an index set $\mathrm{J}$ that depends on the decomposition level.

c) For each subband (here, take d for example), estimate the local variance for each wavelet coefficient using MAP estimation. As to local, we use four sizes of a square $\mathrm{D} \times \mathrm{D}$ neighborhood $\mathrm{E}$, where $\mathrm{D} \in\{3,5,7,9\}$.

$$
\widehat{\delta}_{D}^{2}(i, j)=\max \left[0, \frac{1}{D^{2}} \sum_{(i, j) \in E}\left(d^{2}(i, j)-\delta_{0}^{2}\right)\right](i, j)
$$

$\hat{\delta}_{D}^{2}$ is the local variance estimation. We take the minimum of the four variances as the final estimate

$$
\widehat{\delta}^{2}(i, j)=\min _{D} \hat{\delta}_{D}^{2}(i, j) D \in\{3,5,7,9\},(i, j) \in J
$$

Perform the same operation for $h$ and $v$ subbands.

d) Obtain the denoised wavelet coefficients using Wiener filter:

$$
d_{d N}(i, j)=\mathrm{d}(\mathrm{i}, \mathrm{j}) \frac{\widehat{\delta}^{2}(i, j)}{\widehat{\delta}^{2}(i, j)+\delta_{0}^{2}}(i, j) \in J
$$

and similarly for $h_{d N}$ and $v_{d N}$.

Based on the denoised wavelet coefficients obtained above (all four levels) and the fourth level low frequency subband, we can recover the denoised data in the current channel by inverse wavelet transform. we get the denoised frame $F_{d N}$. As a result, the extracted noise from frame $\mathrm{F}$ is:

$$
\mathrm{N}=\mathrm{F}-F_{d N}
$$
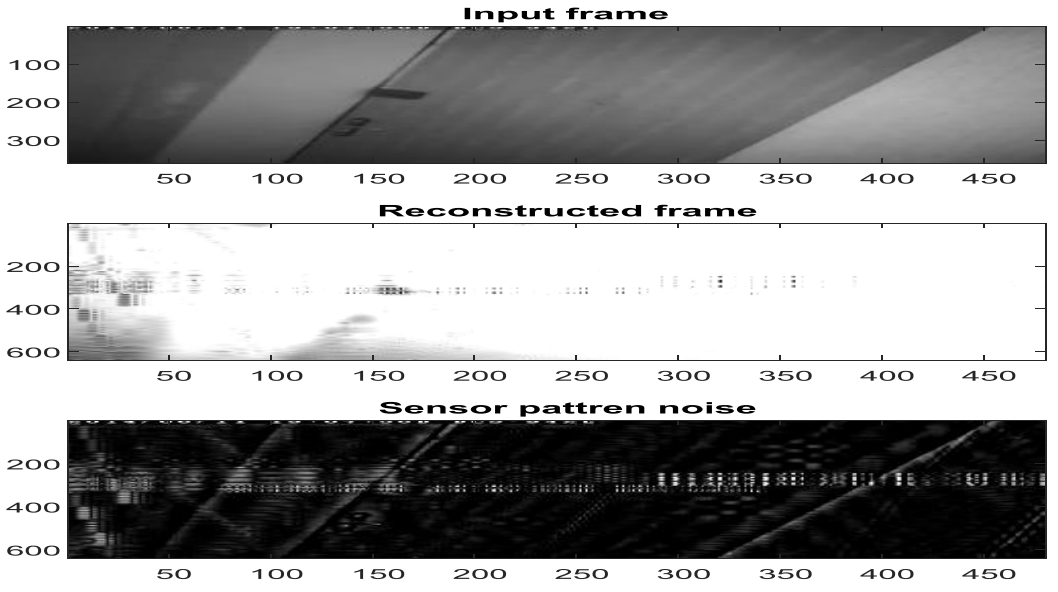

Figure 3.3 Results D-Link DCS-942L camera frame

4. Posterior based source video identification using GMM

In recent application Gaussian Mixture Models (GMM) are used for feature extraction. They are commonly used to represents the continuous probability distribution of measurements. So, it is basically a parametric probability density Model. Its function is represented as a weighted sum of component densities [24]. The parameters are estimated using the iterative Expectation-Maximization (EM) algorithm. Then Maximum A Posteriori (MAP) estimation i.e.maximum likelihood estimation is done for test data with training data formed earlier.

In equation (4.5) below, A Gaussian mixture model is represented.

$$
\mathrm{p}(\mathrm{x} \mid \lambda)=\sum_{i=1}^{M} w_{i} g\left(x \mid \mu_{i}, \Sigma i\right)
$$

where, $\mathrm{x}$ is a D-dimensional continuous-valued data vector, $\mathrm{Wi}, \mathrm{i}=1, \ldots, \mathrm{M}$, are the mixture weights, and $\mathrm{g}(\mathrm{x} \mid$ $\left.\mu_{i}, \Sigma_{i}\right), \mathrm{i}=1, \ldots, \mathrm{M}$, are the component Gaussian densities.

The D-variate Gaussian function is given in form of eq. (3.6),

$$
\mathrm{g}\left(\mathrm{x} \mid \mu_{i}, \Sigma_{i}\right)=\frac{1}{(2 \pi)^{D / 2}|\Sigma|^{1 / 2}} \exp \left\{-\frac{1}{2}\left(x-\mu_{i}\right) \sum_{i}^{-1}\left(X-\mu_{i}\right)\right\}
$$

Here mean vector is represented by $\mu$ i and covariance matrix by $\sum i$.

The mixture weights satisfy the constraint that

$$
\sum_{i=1}^{M} w_{i}=1
$$




\section{IJIREEICE \\ International Journal of Innovative Research in Electrical, Electronics, Instrumentation and Control Engineering \\ ISO 3297:2007 Certified \\ Vol. 5, Issue 5, May 2017}

The Complete Gaussian Mixture model will have mean vectors, covariance matrices and mixture weights from all component densities. Large class of sample distributions can easily be represented by GMMs. Thus, GMMs often used in different mean's data. The arbitrarily shaped densities can be smoothened by GMMs. GMMs is best known for this ability. Feature distributions are represented by classical uni- Gaussian model. The model requires a position vector and covariance matrix generally an elliptic shape. A discrete set of characteristic templates are represented using nearest neighbor model [25]. A GMM acts as a hybrid of the classical uni- Gaussian model and nearest neighbor model. A GMM is hybrid of two models. It uses a discrete set of Gaussian functions. GMMs will have their own mean and covariance matrix. This gives a better modeling capability.

\section{Maximum Likelihood Parameter Estimation}

The first thing is to estimate the parameters of the GMM, $\lambda$. These parameters must best match to given training vectors and a GMM configuration. The Parameters can be calculated using different methods. By far the most popular and well-established method is maximum -likelihood (ML) estimation [26].

In ML estimation model parameters are searched which will match to maximum extend with the GMM trained data set. Suppose there are training Vectors $\mathrm{X}=\left\{x_{1}, \ldots \ldots, x_{T}\right\}$. These Training vector are arranged in T Sequence. They are independent of each other. The independence assumption could not be true in all cases but is needed to assume true as to trace a problem. It can be written as,

$$
\mathrm{p}(\mathrm{X} \mid \lambda)=\sum_{t=1}^{T} p\left(X_{t} \mid \lambda\right)
$$

The function of the parameters $\lambda$ is a non-linear function. The maximum value of parameters could not be obtained directly. However, use a special case of the expectation-maximization (EM) algorithm iteratively [26]. The basic idea of the EM algorithm is the repetition of a process until some convergence threshold is reached.

$$
\mathrm{p}(\mathrm{X} \mid \bar{\lambda}) \geq \mathrm{p}(\mathrm{X} \mid \lambda)
$$

Begin with an initial model $\lambda$, to estimate a new model $\bar{\lambda}$. In next iteration, this new model becomes the initial model. Binary VQ (Vector Quantization) estimation is used for the initial model. The following re-estimation formulas are used for each EM estimation

Means

$$
\bar{\mu}_{i}=\frac{\sum_{t=1}^{T} \operatorname{Pr}\left(i \mid X_{t}, \lambda\right) x_{t}^{2}}{\sum_{t=1}^{T} \operatorname{Pr}\left(i \mid X_{t}, \lambda\right)}-\mu_{i}^{2}
$$

Variances (Diagonal Covariance)

$$
\sigma_{i}^{-2}=\frac{\sum_{t=1}^{T} \operatorname{Pr}\left(i \mid X_{t}, \lambda\right) x_{t}^{2}}{\sum_{t=1}^{T} \operatorname{Pr}\left(i \mid X_{t}, \lambda\right)}-\mu_{i}^{2}
$$

Mixture Weights

$$
\overline{\mathrm{w}_{\mathrm{i}}}=\frac{1}{\mathrm{~T}} \sum_{\mathrm{t}=1}^{\mathrm{T}} \operatorname{Pr}\left(\mathrm{i} \mid \mathrm{X}_{\mathrm{t}}, \lambda\right)
$$

The posteriori probability for component $\mathrm{i}$ is given by

$$
\operatorname{Pr}\left(\left(\mathrm{i} \mid \mathrm{X}_{\mathrm{t}}, \lambda\right)=\frac{\mathrm{w}_{\mathrm{i}} \mathrm{g}\left(\mathrm{X}_{\mathrm{t}} \mid \mu_{\mathrm{i}}, \Sigma_{\mathrm{i}}\right)}{\sum_{\mathrm{k}=1}^{\mathrm{M}} \mathrm{w}_{\mathrm{k}} \mathrm{g}\left(\mathrm{X}_{\mathrm{t}} \mid \mu_{\mathrm{k}}, \Sigma_{\mathrm{k}}\right)}\right.
$$

In brief first GMM model has been generated for all camera classes and then query frame has been checked for source using posterior matching.

\section{EXPERIMENTAL SETUP}

To implement the algorithm formed we have used MATLAB tool. Eight different wireless camera videos are collected and a trained data set is developed. Experimental tests are conducted 160 times i.e. is 20 times for each video to determine the performance of SPN with correlation comparison and SPN with Gaussian Mixture Model.

\section{A. Result Analysis Parameters}

Precision and recall are the basic measures used in evaluating search strategies. As shown in the first two figures on the left, these measures assume: There is a set of records in the database which is relevant to the search topic Records are assumed to be either relevant or irrelevant (these measures do not allow for degrees of relevancy). 
Vol. 5, Issue 5, May 2017

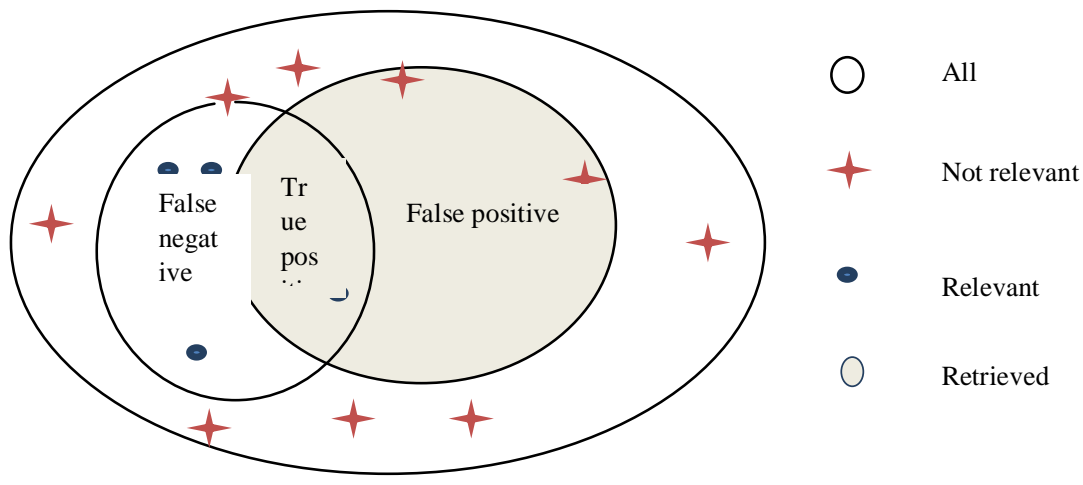

Figure 4.1 Relevant records and Irrelevant records

The actual retrieval set may not perfectly match the set of relevant records. Recall ratio is the ratio of the number of relevant records retrieved to the total number of relevant records in the database. It is usually expressed as a percentage. Formula in terms of true positive and false negative

$$
\text { Recall }- \text { Ratio }=\frac{\mathrm{TP}}{\mathrm{TP}+\mathrm{FN}}
$$

Precision ratio is the ratio of the number of relevant records retrieved to the total number of irrelevant and relevant records retrieved. It is usually Expressed as a percentage.

$$
\text { Precision }- \text { Ratio }=\frac{\mathrm{TP}}{\mathrm{TP}+\mathrm{FP}}
$$

B. Performance evaluation using Correlation coefficient

The noise extraction process will repeat for a sequence of frames from the same video. For source identification, we calculate the sensor pattern noise of the video to be identified (noted $\mathrm{asN}_{\mathrm{v}}$ ), and compare it with the sensor pattern noise extracted from the camera (camera reference pattern for short, noted as $\mathrm{N}_{\mathrm{c}} \mathrm{using}$ the metric of correlation coefficient:

Table 4.1 Recall ratio and precision ratio using correlation coefficient for 20 random tests when taking threshold as .9

\begin{tabular}{|l|l|l|l|l|l|}
\hline Camera & True positive & False positive & False Negative & Recall Ratio & Precision ratio \\
\hline AXIS M1011 & 17 & 4 & 3 & .85 & .8095 \\
\hline D-Link DCS-942L & 18 & 5 & 2 & .90 & .7826 \\
\hline Foscam FI8910W & 15 & 4 & 5 & .75 & .7895 \\
\hline smiledrive Panoramic 360 & 16 & 2 & 4 & .80 & .89 \\
\hline TP-Link NC220 & 18 & 6 & 2 & .90 & .90 \\
\hline TRENDnet TV-IP672W & 14 & 3 & 6 & .70 & .8235 \\
\hline WVC80N & 16 & 4 & 4 & .80 & .80 \\
\hline ZVision AHD 1.3 MP & 15 & 3 & 5 & .75 & .8333 \\
\hline
\end{tabular}

C. Performance Evaluation using GMM posterior Method

Table 4.2 Recall ratio and precision ratio using posterior matching of GMM model

\begin{tabular}{|l|l|l|l|l|l|}
\hline \multicolumn{1}{|c|}{ Camera } & True Positive & False Positive & False Negative & Recall Ratio & Precision ratio \\
\hline AXIS M1011 & 18 & 0 & 2 & .90 & 1 \\
\hline D-Link DCS-942L & 20 & 0 & 0 & 1 & 1 \\
\hline Foscam FI8910W & 20 & 1 & 0 & 1 & .9524 \\
\hline smiledrive Panoramic 360 & 20 & 1 & 0 & 1 & .9524 \\
\hline TP-Link NC220 & 18 & 2 & 2 & .90 & .90 \\
\hline TRENDnet TV-IP672W & 19 & 0 & 1 & .95 & 1 \\
\hline WVC80N & 20 & 1 & 0 & 1 & .9524 \\
\hline ZVision AHD 1.3 MP & 19 & 1 & 1 & .95 & .95 \\
\hline
\end{tabular}




\section{International Journal of Innovative Research in} Electrical, Electronics, Instrumentation and Control Engineering

\section{ISO 3297:2007 Certified}

Vol. 5, Issue 5, May 2017

Mixture Models are a type of density model that comprise a number of component functions, usually Gaussian. These functions are combined to provide a multimodal density. They are employed to model the texture vectors of chosen

$$
\operatorname{corr}\left(\mathrm{N}_{\mathrm{v}}, \mathrm{N}_{\mathrm{c}}\right)=\frac{\left(\mathrm{N}_{\mathrm{v}}-\overline{\mathrm{N}_{\mathrm{v}}}\right)\left(\mathrm{N}_{\mathrm{c}}-\overline{\mathrm{N}_{\mathrm{c}}}\right)}{\left\|\mathrm{N}_{\mathrm{v}}-\overline{\mathrm{N}_{\mathrm{v}}}\right\|\left\|\mathrm{N}_{\mathrm{c}}-\overline{\mathrm{N}_{\mathrm{c}}}\right\|}
$$

Calculating $\mathrm{N}_{c}$ is relatively easy because the source camera is typically accessible to law enforcers. We can use it to take a video (or multiple videos) with sufficient length and high quality, and thus derive the $\mathrm{N}_{\mathrm{c}}$ accurately frames in order to perform tasks for final classification. After generating these models, every frames sensor pattern noise has been compared with all the GMM's using posterior maximum log likelihood, which puts every frame to the closest texture Gaussian model. The results by posterior matching have been shown below.

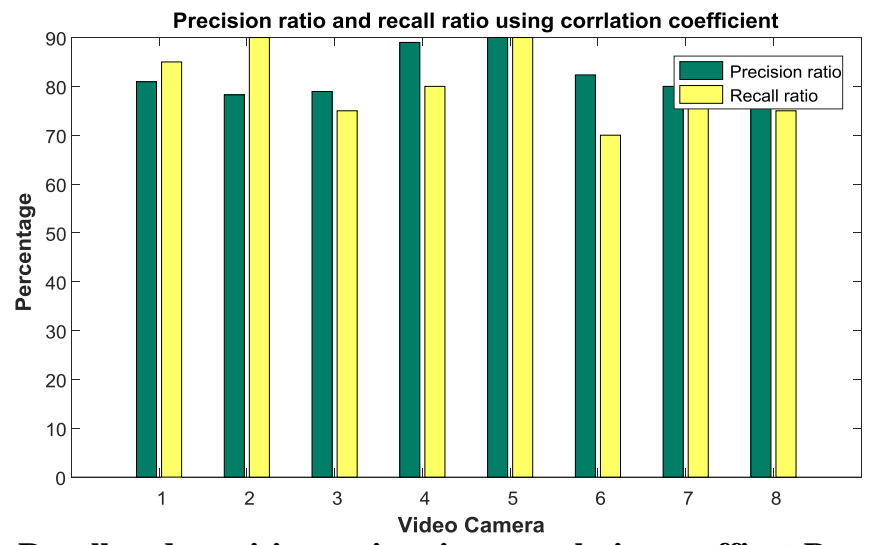

Figure 4.2 Recall and precision ratio using correlation coeffient Based matching

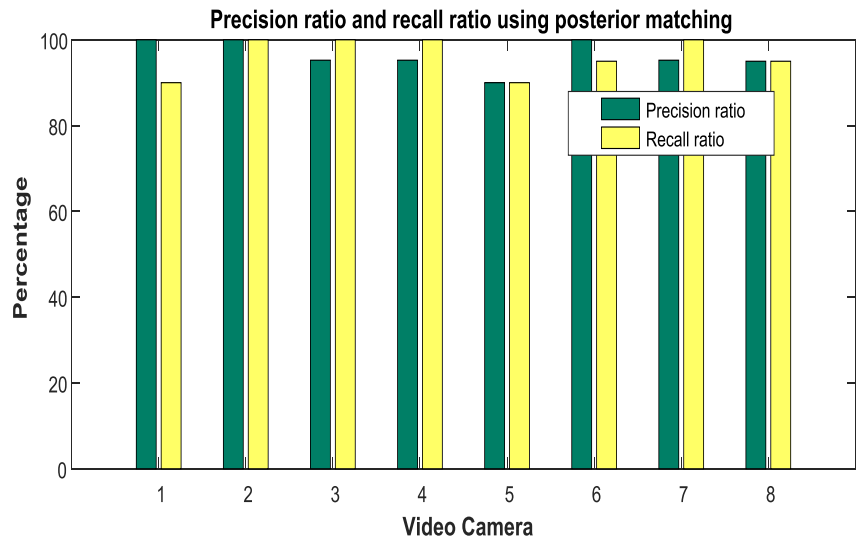

Figure 4.3 Recall and precision ratio using posterior log liklihood Based matching

\section{CONCLUSION}

The sensor pattern noise method exploits the inherent fingerprint of camera sensor and is universally applicable. This method has the best performance reported so far among the existing methods and only method effective in lossy wireless network. It is applicable on different size video. The requirement of random frames is very less as compared to existed work. In correlation coefficient based identification method, there is requirement of more frames for accurate result. In this, the algorithm has been tested for minimum five random frames for generating Gaussian mixture models from evaluated sensor pattern noise of individual frame. The complexity has reduced as working on single gray channel instead of three color channels. Gaussian Mixture Models help to train pre-set video once and then just need query frames to be tested which will save time of testing but in correlation coefficient method will require to be developed each time which make it complex and require time. The method becomes more effective by having a strong train database.

As no research work is ever complete. Every research study is based upon certain assumptions and approximations that leave us scope for future studies. The proposed work can be carried forward to make it more effective when dealing with illumination factor and to work with advanced security attacks in wireless network. 


\section{IJIREEICE

\section{REFERENCES}

[1] S. Chen, A. Pande, K. Zeng, and P. Mohapatra, January 2015 , "Live Video Forensics: Source Identification in Lossy Wireless Networks,” IEEE Transactions on Information Forensics and Security, vol.10, NO.1, pp. 28 - 39.

[2] M. Chen, J. Fridrich, M. Goljan, and J. Lukáš, February 2007 "'Source digital camcorder identification using sensor photo response nonuniformity," Proceeding SPIE, Security, Steganogr., Watermarking Multimedia Contents IX, vol. 6505, no. 1, pp. 65051G.

[3] F. Lefebvre, B. Chupeau, A. Massoudi, and E. Diehl, Februrary 2009, "Image and video fingerprinting: Forensic application s," Proceeding SPIE, Media Forensics Security, vol. 7254, pp. 1-9, Art. ID 725405.

[4] Y. Su, J. Xu, and B. Dong, October 2009, "A source video identification algorithm based on motion vectors" in Proceeding 2nd International Workshop Computer Science Engineering, Vol. 2., pp. 312-316.

[5] Z. J. Geradts, J. Bijhold, M. Kieft, K. Kurosawa, K. Kuroki, and N. Saitoh, February 2001, "Methods for identification of images acquired with digital cameras," Proceeding SPIE, Enabling Technolology Law Enforcement Security, vol. 4232, pp. 505-512.

[6] J. Lukáš, J. Fridrich, and M. Goljan, June 2006, "Digital camera identification from sensor pattern noise" IEEE Transactions Information Forensics Security, vol. 1, no. 2, pp. 205-214.

[7] M. Kharrazi, H. T. Sencar, and N. Memon, October 2004, "Blind source camera identification," in Proceedings IEEE International Conference Image Processing, Singapore, pp. 709-712.

[8] O. Celiktutan, I. Avcibas, B. Sankur, and N. Memon, 2005, "Source cell-phone identification," in Proceesing International Conference Advance Computer Communication, Tamil Nadu, India.

[9] K. S. Choi, E. Y. Lam, and K. K. Y. Wong, 2006, "Automatic source camera identification using the intrinsic lens radial distortion," Optics Express, vol. 14, no. 24, pp. 11551-11565.

[10] A. C. Popescu, 2004, "Statistical tools for digital image forensics," Ph.D. dissertation, Department of Computer Science., Dartmouth College, Hanover, NH, USA.

[11] C.-T. Li, "Source camera identification using enhanced sensor pattern noise, June 2010," IEEE Transactions Information Forensics Security, vol. 5, no. 2, pp. 280-287.

[12] K. Kurosawa, K. Kuroki, and N. Saitoh, 1999, "CCD fingerprint method-Identification of a video camera from videotaped images," in Proceeding IEEE International Conference of Image Processing, pp. 537-540.

[13] Zeno J Geradts, Jurrien Bijhold, Martijn Kie, Kenro Kuroki, and Naoki Saitoh, 2001,“ Methods for identification of images acquired with digital cameras,".

[14] W. van Houten and Z. Geradts, 2009, "Source video camera identification for multiply compressed videos originating from YouTube,” Digital Investigation., vol. 6, nos. 1-2, pp. 48-60.

[15] D.-K. Hyun, C.-H.Choi, and H.-K. Lee, 2012, "Camcorder identification for heavily compressed low resolution videos" in Computer Science and Convergence, Amsterdam, The Netherlands: Springer-Verlag, vol. 114, pp. 695-701.

[16] Ambuj Mehrish, A.V Subramanyam and Sabu Emmanuel, 2015, " Sensor Pattern Noise Estimation Using Probabilistically Estimated RAW Values" in IEEE Signal Processing Letters.

[17] Xufeng Lin and Chang-Tsun Li, March 2016, "Enhancing Sensor Pattern Noise via Filtering Distortion Removal”, IEEE Signal Processing Letters, VOL. 23, NO. 3.

[18] Ashref Lawgaly, and Fouad Khelifi, 2016, "Sensor Pattern Noise Estimation Based on Improved Locally Adaptive DCT Filtering and Weighted Averaging for Source Camera Identification and Verification", IEEE Transactions on Information Forensics And Security.

[19] Yuping Duan, Dalel Bouslimi, Guanyu Yang, and Huazhong Shu and Gouenou Coatrieux, 2016, "Computed Tomography Image Origin Identification based on Original Sensor Pattern Noise and 3D Image Reconstruction Algorithm Footprints", IEEE Journal of Biomedical and Health Informatics.

[20] S. Fogie. , 2007, (“Abusing and Misusing Wireless Cameras" [Online].

[21] S. Chen, A. Pande, K. Zeng, and P. Mohapatra, April 2013, "Video source identification in lossy wireless networks," in Proceeding IEEE INFOCOM, Turin, Italy, pp. 215-219.

[22] Amit Pande, Shaxun Chen, Prasant Mohapatra and Joseph Zambreno, January 2014, "Hardware Architecture for Video Authentication Using Sensor Pattern Noise", IEEE Transactions on circuits and systems for video technology, Vol. 24, No. 1.

[23] H. Zhang, A. Nosratinia and R. O. Wells, 2000, "Image denoising via wavelet-domain spatially adaptive FIR Wiener filtering," IEEE International Conference on Acoustics, Speech, and Signal Processing. Proceedings (Cat. No.00CH37100), Istanbul, pp. 2179-2182 vol.4.

[24] Reynolds, Douglas. "Gaussian Mixture Models," MIT Lincoln Laboratory, 244 Wood St., Lexington, MA 02140, USA: 1-5. Print.

[25] S. Dempster, A., Laird, N., Rubin, D, 1997, “ Maximum Likelihood from Incomplete Data via the EMAlgorithm. ," Journal of the Royal Statistical Society 39(1),pp.1-38. 